\title{
Sensitivity, Specificity, and Accuracy of Color Doppler Ultrasonography for Diagnosis of Retinal Detachment
}

\author{
Mohammadreza Akhlaghi ${ }^{1}$, MD; Masoomeh Zarei ${ }^{1}$, MD; Majid Ziaei ${ }^{2}$, MD; Mohsen Pourazizi ${ }^{1,3}$, MD \\ ${ }^{1}$ Department of Ophthalmology, Isfahan Eye Research Center, Isfahan University of Medical Sciences, Isfahan, Iran \\ ${ }^{2}$ Nafis Private Imaging Center, Isfahan, Iran \\ ${ }^{3}$ Pediatric Inherited Diseases Research Center, Research Institute for Primordial Prevention of Non-Communicable Disease, \\ Isfahan University of Medical Sciences, Isfahan, Iran \\ ORCID: \\ Mohammadreza Akhlaghi: https://orcid.org/0000-0002-5395-1809 \\ Mohsen Pourazizi: https://orcid.org/0000-0002-9714-8209
}

\section{Abstract}

Purpose: This study evaluated the sensitivity, specificity, and diagnostic accuracy of Color Doppler Ultrasonography (CDUS) in patients with suspected retinal detachment (RD) who underwent surgery.

Methods: In this prospective, observational clinical study, 65 eyes of 65 consecutive patients with suspected RD with opaque media were included. Following a standardized protocol, CDUS of the retina of the affected eye was performed. The sensitivity, specificity, and diagnostic accuracy of CDUS were determined and compared to the findings during surgery.

Results: The mean age of patients (18 men and 47 women) was 52.36 years (range: 8-77 years). The sensitivity, specificity, and overall accuracy of ocular CDUS were $91.3 \%, 88.1 \%$, and $89.2 \%$, respectively. The false-negative rate (negative CDUS images but presence of RD at operation) was 3.1\% (2/65) and the false-positive rate (positive CDUS images but absence of RD at operation) was 7.7\% (5/65).

Conclusion: CDUS of the retina could be considered as a promising tool in the diagnosis of RD in patients with opaque media.

Keywords: Color Doppler Ultrasonography; Retina; Retinal Detachment

J Ophthalmic Vis Res 2020; 15 (2): 166-171

Correspondence to:

Mohsen Pourazizi, MD. Department of Ophthalmology, Isfahan University of Medical Sciences, Isfahan 81496, Iran.

E-mail: m.pourazizi@yahoo.com

Received: 26-08-2017 Accepted: 23-09-2019

\section{Access this article online}

Website:

https://knepublishing.com/index.php/JOVR

DOI:

10.18502/jovr.v15i2.6733

\section{INTRODUCTION}

Retinal detachment (RD) is a serious eye disease with the potential risk of blindness. It requires

This is an open access journal, and articles are distributed under the terms of the Creative Commons Attribution-NonCommercial-ShareAlike 4.0 License, which allows others to remix, tweak, and build upon the work non-commercially, as long as appropriate credit is given and the new creations are licensed under the identical terms.

How to cite this article: Akhlaghi M, Zarei M, Ziaei M, Pourazizi M. Sensitivity, Specificity, and Accuracy of Color Doppler Ultrasonography for Diagnosis of Retinal Detachment. J Ophthalmic Vis Res 2020;15:166-171. 
immediate management to prevent permanent vision loss. ${ }^{[1,2]}$ Accurate diagnosis of RD may be difficult in some cases and is important as it affects the prognosis and treatment plan. ${ }^{[3]}$

It is recommended that ophthalmologists rely on their clinical skills, including indirect ophthalmoscopy and biomicroscopic examination of the fundus with slit lamp, for the diagnosis of RD in suspected cases. ${ }^{[1]}$

In the presence of dense media opacities (e.g., corneal edema, hyphema, cataract, vitreous hemorrhages) with clinical suspicion of RD, the diagnosis may not be accurate, and poor visual prognosis can be expected if the ophthalmologists wait for the media to clear. ${ }^{[4,5]}$ Moreover, the decision to perform surgery in all clinically suspected cases poses additional costs on patients and the healthcare system. Therefore, alternative diagnostic modalities, such as ocular ultrasound, are required in this clinical situation. ${ }^{[6]}$ Gray-scale ultrasound can identify RD in suspected cases with dense media opacities. ${ }^{[7]}$

Although gray-scale ultrasound can differentiate $\mathrm{RD}$ from other membranous structures in the presence of opaque media, it has some limitations that may lead to a diagnostic dilemma. ${ }^{[6,8]}$ Differentiating individual retinal layers is not possible on grayscale ultrasound so it may be difficult to accurately differentiate retinoschisis from $\mathrm{RD}^{\left[{ }^{[9]}\right.}$ The posterior hyaloid membrane or central vitreous gel are not usually attached to the optic disc and are visualized as structures with weaker echogenicity and more variable thickness, with a greater mobility than RD. However, differentiating RD from those structures in the posterior segment is difficult in cases of shallow or localized RD. ${ }^{[10,11]}$

In such cases, color Doppler ultrasound (CDUS) can play an important role in the diagnosis of RD by demonstrating blood flow in the detached retina. CDUS helps ophthalmologists examine the retinal blood flow, even in the presence of dense ocular opacities preventing a direct view of the posterior segment. ${ }^{[12]}$ The detached retina is seen on CDUS as a curvilinear structure in the vitreous cavity with blood flow. ${ }^{[8,12]}$ Therefore, CDUS can play an additional and more reliable role in the diagnosis through visualization of flow signals in the detached retina. ${ }^{[8]}$

However, despite CDUS having several potential benefits, a limitation of the application of CDUS in the diagnosis of RD is that the evidence of its diagnostic accuracy is scattered and limited. The aim of this study was to evaluate the clinical utility and diagnostic value of CDUS in the detection of $\mathrm{RD}$ in patients with dense ocular opacities.

\section{METHODS}

\section{Patients and Study Design}

This prospective, observational clinical study was approved by the Ethics Committee of Isfahan University of Medical Sciences. The study protocol adhered to the tenets of the Declaration of Helsinki. Signed informed consent was obtained from participants prior to the study. We enrolled 65 eyes of 65 patients with opaque media who were candidates for ocular surgery to clear the media and repair RD if present (pars plana vitrectomy +/phacoemulsification, with or without corneal graft, etc.). Patients who were inoperable because of any medical, surgical, or general condition and those with a past or current history of tumors in the vitreous cavity were excluded.

\section{Doppler Ultrasound Imaging}

On the day of enrollment, CDUS of the eye was performed for all individuals by an expert radiologist trained in ultrasound of the retina using a CDUS unit and 13-MHz linear array transducer, Medison V20 (Medison Co. Ltd., Seoul, Korea). The evaluation techniques have been described previously. ${ }^{[8]}$ Patients were examined in the supine position to avoid any pressure on the eye. Sterile coupling gel was applied to the closed eyelids, with the examiner's hand resting on the orbital margin to minimize pressure on the globe, and color flow images were obtained. ${ }^{[8]}$

RD was described as visualization of a flow signal (presence of vascularity) along the detached retina, while vitreous membranes were described as lack of vascularity. ${ }^{[10]}$

\section{Reference Standard}

Fundus examination during surgery was considered as the gold standard for the diagnosis of RD. The final clinical diagnosis of RD was made by two expert ophthalmologists (first and second authors) during surgery. 


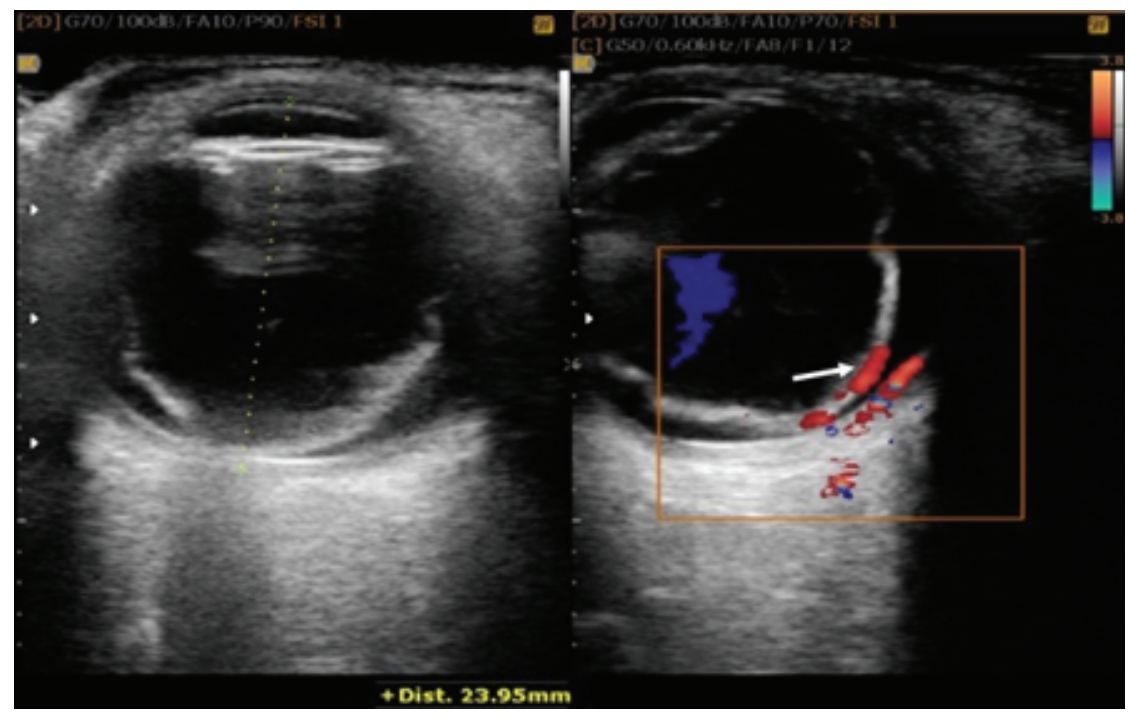

Figure 1. (Left) The funnel-shaped membrane has been marked on the gray-scale B scan. (Right) The figure shows arterial flow and retinal detachment.

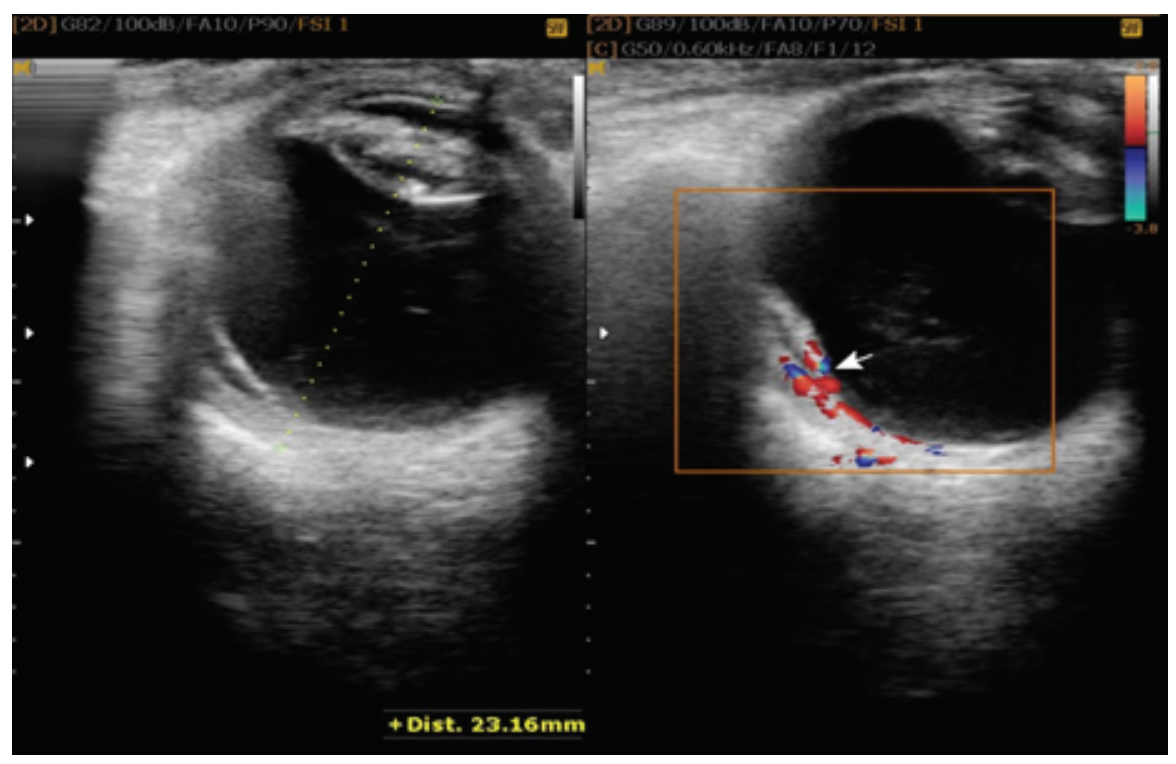

Figure 2. (Left) The gray-scale image of the horizontally scanned eye demonstrates central partial retinal detachment. (Right) Color Doppler ultrasound reveals thick linear and spotty color signals.

\section{Statistical Analysis}

Statistical analyses were performed by a statistician using the SPSS software, version 16.0 (SPSS, Chicago, IL, USA). Sensitivity and specificity were calculated for diagnostic CDUS, with the final clinical diagnosis during the operation as the reference standard. Sensitivity was calculated as the proportion of patients with actual RD who had an abnormal retina on CDUS. Specificity was calculated as the proportion of patients with no actual $\mathrm{RD}$ who had a normal retina on CDUS. Accuracy was calculated as the proportion of patients whose RD status was correctly predicted using ultrasound.

\section{RESULTS}

In this study, we performed CDUS for 65 eyes of 65 patients, including 18 men and 47 women, suspected with RD. The mean age of patients was 52.36 years (range: 8-77 years). Vitreous hemorrhage, cataract, total hyphema, and corneal opacity were detected in 44 (67.7\%), 12 (18.58\%), 6 (9.2\%), and 3 eyes, respectively. 


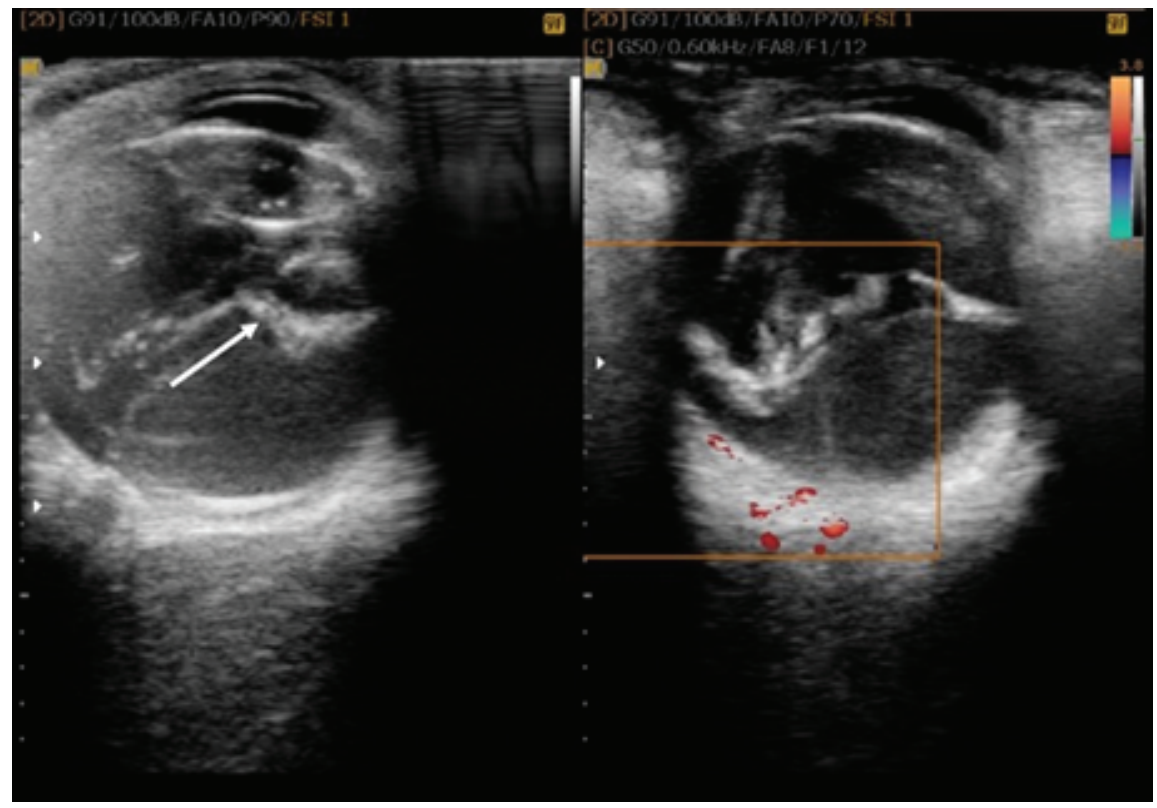

Figure 3. Color Doppler ultrasonography shows no flow in the membrane seen in the left figure, ruling-out RD. The avascular membrane has been marked in the right figure.

Of the 65 patients, RD was diagnosed in 26 (40\%) patients on CDUS and in 23 (35.4\%) patients during vitrectomy. As for the type of RD, all patients had rhegmatogenous RD.

The sensitivity, specificity, and overall accuracy of ocular CDUS were 91.3\%, 88.1\%, and $89.2 \%$, respectively. The false-negative rate (negative with CDUS but positive with operation) was 3.1\% (2/65) and the false-positive rate (positive with CDUS but negative with operation) was 7.7\% (5/65). False-positive cases included four cases of severe neovascular membrane in proliferative diabetic retinopathy and one case of posterior vitreous detachment.

\section{DISCUSSION}

The current study demonstrated that CDUS is sensitive and specific for the diagnosis of RD in patients with dense ocular opacities. In most cases, gray-scale B-mode ultrasonography allows differentiation of a total RD from a vitreous membrane; however, this differentiation may be challenging in some situations. ${ }^{[4,13,14]}$ Cases of partial RD and vitreous membrane can share similar ultrasonographic features. ${ }^{[4,5,14]}$ In patients with atypical findings on gray-scale ultrasound of shallow RD, CDUS can play an additional and more reliable role in the diagnosis by enabling detection of blood flow in the detached retina. ${ }^{[13,15]}$
Advantages of performing CDUS to diagnose RD are that CDUS can be a quick, noninvasive, and safe method for detecting total and partial RDs. CDUS enables ophthalmologists to examine ocular blood flow, even in the presence of dense ocular opacities preventing a direct view to the posterior segment of the eye. ${ }^{[12]}$

A previous study at Isfahan Eye Research Center by Ghanbari et al indicated the diagnostic data of gray-scale sonography as follows: sensitivity, 87.5\%; specificity, $64.5 \%$; and accuracy, $72.4 \%{ }^{[16]}$

In the current study performed at the same center, the sensitivity, specificity, and overall accuracy of ocular CDUS compared to surgical findings were $91.3 \%, 88.1 \%$, and $89.2 \%$, respectively. These differences can be explained by limitations of gray-scale ultrasound in detection of RD as previously mentioned. There is no quick, noninvasive, and safe gold standard for the diagnosis of RD. Although Bscan has the aforementioned characteristics, there are some limitations. ${ }^{[8,16]}$

Studies examining changes in ocular blood flow velocities in RD are limited. ${ }^{[10]}$ Ido et al found the usefulness of CDUS in the diagnosis of RD in the presence of hazy media. ${ }^{[10]}$ Similar to ours, in their study, the absence or presence of RD was confirmed during surgery. Their study on 33 consecutive patients demonstrated a sensitivity of $92.3 \%$, a specificity of $100 \%$, a positive predictive value of $100 \%$, a negative predictive value of $93.3 \%$, 
and an accuracy of $96.3 \%$. In their study, all patients with blood flow on CDUS were confirmed to have RD during surgery. ${ }^{[10]}$

In the study by Han et al, CDUS showed a color signal in approximately $60 \%$ of RD cases. ${ }^{[8]}$ Wong et al reported the sensitivity of CDUS to be $100 \%{ }^{[17]}$ The sensitivity and specificity of ocular CDUS were both approximately $90 \%$ in our study. The difference between these results can be explained by certain factors, including the type of RD, duration of detachment, coexisting pathologies, etc.

The ability of CDUS to demonstrate flow in vascular structures and subsequently yield the diagnosis of RD in patients with dense ocular opacities depends on factors including flow velocity, vessel size, depth of the lesion, scanner sensitivity, and operator control. ${ }^{[8]}$ Therefore, the sensitivity, specificity, and accuracy can be affected. ${ }^{[8]}$ Most longstanding RDs are peripheral, not involving the posterior pole. A reason for the difference in results of similar studies in this field is the presence of longstanding RD. CDUS has a lower ability to detect blood flow in the retinal periphery. This may be a possible explanation for the false-negatives of CDUS. ${ }^{[8]}$

Despite several potential benefits, the interobserver variability could be a possible explanation for CDUS not being routinely used in ophthalmic practice. ${ }^{[18]}$ To increase the detectability of Doppler signals, several kinds of ultrasound contrast agents can be used. In the study by Han et al, the sensitivity of CDUS to detect flow in RD increased from approximately $60 \%$ to 90\% after intravenous contrast administration. ${ }^{[8]}$ Although a highly accurate diagnosis is achieved using contrast-enhancing agents, the procedure would be invasive.

In our study, two patients who were negative for $\mathrm{RD}$ on CDUS were diagnosed with RD based on surgical data. We did not find any meaningful difference between the characteristics of these patients and others, for example, regarding the type of RD. Negative results on CDUS but positive results during operation may occur in the presence of any ischemic event in the retinal vessels, including arterial and venous occlusions.

Of the false-positive cases in the current study, four cases were of severe neovascular membrane in proliferative diabetic retinopathy and one case was of posterior vitreous detachment. There were no cases of traction RD.
The overall accuracy of CDUS was approximately $90 \%$. In the study by Wong et al, ${ }^{[1]}$ all patients with RD were diagnosed using CDUS, consistent with our results.

There are some limitations in the current study, including the relatively small sample size. Relative afferent pupillary defects were not recorded in this study, and their relationship with other findings was not evaluated. Failure to use contrast-enhanced CDUS is another shortcoming of the current study. The lower ability of CDUS to detect blood flow in the retinal periphery is an inherent limitation of the technique. The prospective nature of this study on the accuracy of CDUS in diagnosing RD in eyes with dense ocular opacities and suspected RD is a strength of the current study.

In conclusion, CDUS helps in distinguishing between RD and vitreous membrane in eyes with opaque media when the results of B-scan sonography are inconclusive. Large, prospective studies are required to confirm the greater accuracy of CDUS compared to other modalities in the diagnosis of RD in these patients.

\section{Financial Support and Sponsorship}

None.

\section{Conflicts of Interest}

There are no conflicts of interest.

\section{REFERENCES}

1. Hollands H, Johnson D, Brox AC, Almeida D, Simel DL, Sharma S. Acute-onset floaters and flashes: is this patient at risk for retinal detachment? JAMA 2009;302:22432249.

2. Jacobsen B, Lahham S, Patel A, Spann S, Fox JC. Retrospective review of ocular point-of-care ultrasound for detection of retinal detachment. West J Emerg Med 2016;17:196-200.

3. Hoogewoud F, Chronopoulos A, Varga Z, Souteyrand G, Thumann G, Schutz JS. Traumatic retinal detachmentthe difficulty and importance of correct diagnosis. Surv Ophthalmol 2016;61:156-163.

4. Dietrich UM. Oead, Part 3: Diagnostic ultrasonography. In: Gelatt KN, editor. Veterinary ophthalmology. 4th ed. Volume 1. Hoboken, NJ: Blackwell Publishing; 2007:507519.

5. Spaulding K. Eye and orbit. In: Penninck D, Anjou MA, editors. Atlas of small animal ultrasonography. Hoboken, NJ: Blackwell Publishing; 2008:49-90. 
6. Kerman BM, Coleman DJ. B-scan ultrasonography of retinal detachments. Ann Ophthalmol 1978;10:903-911.

7. Blaivas M, Theodoro D, Sierzenski PR. A study of bedside ocular ultrasonography in the emergency department. Acad Emerg Med 2002;9:791-799.

8. Han SS, Chang SK, Yoon JH, Lee YJ. The use of contrastenhanced color Doppler ultrasound in the differentiation of retinal detachment from vitreous membrane. Korean $\mathrm{J}$ Radiol 2001;2:197-203.

9. Agarwal A, Fan S, Invernizzi A, Do DV, Nguyen QD, Harms NV, et al. Characterization of retinal structure and diagnosis of peripheral acquired retinoschisis using high-resolution ultrasound B-scan. Graefes Arch Clin Exp Ophthalmol 2016;254:69-75.

10. Ido M, Osawa S, Fukukita M, Sugimoto M, Wakitani Y, Ito Y, et al. The use of colour Doppler imaging in the diagnosis of retinal detachment. Eye 2007;21:1375-1378.

11. Kaiser HJ, Schotzau A, Flammer J. Blood-flow velocities in the extraocular vessels in normal volunteers. Am J Ophthalmol 1996;122:364-370.

12. Dimitrova $\mathrm{G}$, Kato S. Color Doppler imaging of retinal diseases. Surv Ophthalmol 2010;55:193-214.
13. Santos L, Capeans C, Gonzalez F, Lorenzo J, Codesido $J$, Salorio MS. Ocular blood flow velocity reduction after buckling surgery. Graefes Arch Clin Exp Ophthalmol 1994;232:666-669.

14. Gonzalez EM, Rodriguez A, Garcia I. Review of ocular ultrasonography. Vet Radiol Ultrasound 2001;42:485495.

15. Stefanczyk L, Orawiec B, Gralek M, Majka R, Niwald A. [Usefulness of color Doppler ultrasonography in diagnosis of retinal detachment]. Klin Oczna 1996;98:287-290.

16. Ghanbari H, Dehghani A, Akhlaghi M, Naderi Bani A, Hoghooghi A. Specifity and sensitivity of ocular sonography in the diagnosis of retinal detachment with vitreous hemorrhage. Bina J Ophthalmol 2008;13:432-436.

17. Wong AD, Cooperberg PL, Ross WH, Araki DN. Differentiation of detached retina and vitreous membrane with color flow Doppler. Radiology 1991;178:429-431.

18. Williamson TH, Baxter GM. Central retinal vein occlusion, an investigation by color Doppler imaging. Blood velocity characteristics and prediction of iris neovascularization. Ophthalmology 1994;101:1362-1372. 